\title{
1 Metagenomes of Red Sea subpopulations challenge the use of 2 morphology and marker genes to assess Trichodesmium diversity

4 Coco Koedooder ${ }^{1,2,3^{*}}$, Etai Landou ${ }^{4}$, Futing Zhang ${ }^{1,2}$, Siyuan Wang ${ }^{1,2}$, Subhajit Basu ${ }^{1,2,5}$, Ilana Berman-Frank ${ }^{6}$, $5 \quad$ Yeala Shaked ${ }^{1,2}$, Maxim Rubin-Blum ${ }^{3}$

$6 \quad{ }^{I}$ The Fredy and Nadine Herrmann Institute of Earth Sciences, Hebrew University of Jerusalem, Jerusalem, Israel

$7 \quad{ }^{2}$ The Interuniversity Institute for Marine Sciences in Eilat, Eilat, Israel

$8 \quad{ }^{3}$ Israel Oceanographic and Limnological Research, Haifa, Israel

$9 \quad{ }^{4}$ Mina and Everard Goodman Faculty of Life Sciences, Bar Ilan University, Ramat Gan, Israel

10 5icrosensor Research Group, Max Planck Institute for Marine Microbiology, Bremen, Germany

$11{ }^{6}$ Dept. of Marine Biology, Leon H. Charney School of Marine Sciences, University of Haifa, Haifa, Israel

12

13

*Correspondence: Coco Koedooder (coco.koedooder@huji.ac.il)

14

15 Keywords: Trichodesmium, genotype, cyanobacteria, morphotype, Red Sea, taxonomy 


\section{Abstract}

18 The bloom forming Trichodesmium are filamentous cyanobacteria of key interest due to their 19 ability to fix carbon and nitrogen within an oligotrophic marine environment. Trichodesmium blooms typically comprise a complex assemblage of subpopulations and colony-morphologies that

21 are predicted to exhibit distinct ecological lifestyles. Here, we assessed the poorly studied diversity 22 of Trichodesmium in the Red Sea, based on metagenome-assembled genomes (MAGs) and hetR 23 gene-based phylotyping.

25 We assembled four non-redundant MAGs from morphologically distinct Trichodesmium colonies 26 (tufts, dense and thin puffs). T. thiebautii (puffs) and T. erythraeum (tufts) were the dominant 27 species within these morphotypes. While subspecies diversity is present for both T. thiebautii and 28 T. erythraeum, a single $T$. thiebautii genotype comprised both thin and dense puff morphotypes, 29 and we therefore hypothesize that the phenotypic variation between these morphologies is likely attributed to gene regulation. Additionally, we found the rare non-diazotrophic clade IV and V

31 genotypes, related to $T$. nobis and T. miru respectively, that likely occurred as single filaments. 32 HetR gene phylogeny indicates that the genotype in clade IV could represent the species $T$. contortum.

35 We further show that hetR phylotyping can overestimate the taxonomic diversity of 36 Trichodesmium, as two copies of the hetR gene were present within $T$. thiebautii genomes, one of 37 which misidentified this lineage as T. aureum. Taken together, our results highlight the importance 38 of re-assessing Trichodesmium taxonomy while showing the ability of genomics to capture the complex diversity and distribution of Trichodesmium populations. 


\section{Introduction}

41 Trichodesmium is a genus of filamentous cyanobacteria known for its ability to form large visible

42 surface blooms in tropical and subtropical regions of the ocean. Having first been described in

431770 by James Cook $^{1}$, Trichodesmium have been extensively studied due to their ability to form

44 blooms of large biomass supporting marine food webs through their nitrogen and carbon fixing

45 capabilities $^{2}$ and subsequent relevance to biogeochemical cycles within oligotrophic marine

46 environments ${ }^{3-5}$.

47

48 Trichodesmium blooms are dynamic over space and time, typically consisting of a complex

49 assemblage of several different subpopulations and colony morphologies that are predicted to

50 exhibit unique ecological lifestyles ${ }^{6-11}$. For example, the comparison of two distinct puff-shaped

51 morphotypes termed 'dense' and 'thin' colonies, isolated from the Red Sea displayed a remarkable

52 heterogeneity in their preference to capture and center dust ${ }^{7}$. While colony morphotypes cannot

53 always be linked to different genotypes or species ${ }^{8,12}$ genomic information regarding

54 Trichodesmium colonies in this region is lacking in comparison to studies conducted in the Atlantic

55 and Pacific Oceans.

56

57 The diversity of Trichodesmium bloom-forming populations was first assessed via colony

58 morphology ${ }^{13}$. With the advent of molecular techniques, the use of single-marker genes

59 represented a more accurate and consistent phylogenetic technique ${ }^{14}$. Marker genes that have been

60 used to assess Trichodesmium diversity include the $16 \mathrm{~S}$ rRNA, hetR, a regulatory gene for the

61 development of heterocysts, and nifH, which encodes the nitrogenase iron protein. The hetR gene 
62 is considered a good marker to assess Trichodesmium diversity as it is more variable (10\%) in comparison to genetic markers such as $16 \mathrm{~S}$ ribosomal RNA $(2-3 \%)$ or $n i f H(2 \%)^{15,16}$.

64

65 Phylogenetic analysis using het $R$ as a marker gene separates Trichodesmium into four distinct

66

67 clades: clade I (T. thiebautii, T. hildebrandtii, T. tenue, T. pelagicum), clade II (T. aureum), clade III (T. erythraeum, T. havanum), and clade IV (T. contortum, T. tenue) $)^{15,17}$. Recently, a fifth clade (clade V) consisting of the non-diazotrophic T. miru was proposed by Delmont (2021). Currently, genomes of clades I, III, (and V) are available, including that of the culturable clade III lineage Trichodesmium erythraeum IMS101 ${ }^{18}$.

To better understand the taxonomic diversity of Trichodesmium in the Red Sea, we isolated colonies from the Gulf of Aqaba and compared the resulting metagenome-assembled genomes (MAGs) to those of other Trichodesmium populations from the Indian, Pacific and Atlantic Oceans. We confirmed the presence of these genomes in the Red Sea based on amplicon sequencing of the hetR gene, and, in light of our findings, evaluated the ability of this marker gene to capture Trichodesmium diversity.

\section{Material and Methods}

\section{Trichodesmium sampling and extraction}

To assess the Trichodesmium population of the Red Sea, Trichodesmium colonies were handpicked and separated into three distinct morphotypes $(\sim 100-200$ colonies each) throughout the winter bloom (November 2020) using a $100 \mu \mathrm{m}$ phytoplankton net at $20 \mathrm{~m}$ depth in the Gulf of Aqaba (Eilat, Israel) $\left(29.56^{\circ} \mathrm{N}, 34.95^{\circ} \mathrm{E}\right)$ (Figure 1). Colonies were washed 3 times in $0.2 \mu \mathrm{m}$ filtered 
85 seawater before being filtered on a $0.2 \mu \mathrm{m}$ PCC filter using a vacuum pump. Filters were frozen

86 in liquid $\mathrm{N}_{2}$ and kept at $-80^{\circ} \mathrm{C}$ until analysis.

87

DNA was extracted from three Trichodesmium samples using the DNAeasy Plant Mini Kit

(Qiagen) following the manufacturer's instructions. Metagenomic libraries were prepared at

HyLabs (Rehovot, Israel) and sequenced with 30-60 million 2 x 150 bp reads on Illumina HiSeq at GENEWIZ (Leipzig, Germany). The metagenomic samples can be found under the accession numbers SRR17940154-6.

\section{Metagenomic analysis}

Trichodesmium metagenome-assembled genomes (MAGs) were binned from assemblies based on the raw reads from this study (PRJNA804487) and those from the previously published metagenomes of Trichodesmium from the Pacific (PRJNA435427; PRJNA358796) and Atlantic (PRJNA330990) Oceans ${ }^{8,19,20}$. Metagenomes were assembled, annotated, and binned using the 
MAGs, including two novel non-diazotrophic genomes ${ }^{6}$, were incorporated in the follow-up analysis. The ANI values for each MAG were analyzed using ANIb module in pyANI ${ }^{33}$.

\section{Amplicon sequencing of the hetR gene}

Trichodesmium samples (tuft and puff morphotypes) for amplicon sequencing of the het $R$ were collected in the Gulf of Aqaba (same location as above) during several blooms (2013-2019). DNA was extracted using the phenol-chloroform method ${ }^{34}$. Partial het $R$ gene sequences $(\sim 355 \mathrm{bp})$ were amplified using the forward primer (hetrR_50F), 5'-ATTGAACCYAAACGGGTT-3' and reverse primer (hetR_381R), 5' CGCTTAATATGTYCTGYCAAAGCTT-3', which were deduced from conserved regions of a Trichodesmium hetR nucleotide sequence alignment. 2 x 250 bp reads were sequenced on Illumina MiSeq, following library preparation at HyLabs (Rehovot, Israel). The het $R$ amplicion samples can be found under the accession numbers SAMN25885796-803. Sequences were merged, denoised and called into amplicon sequence variants (ASVs) using DADA2 in Qiime2 $2^{35,36}$. The ASVs were clustered into five OTUs at 98\% similarity for further downstream analyses using the VSEARCH consensus taxonomy classifier ${ }^{37}$.

\section{Trichodesmium phylogeny}

We constructed multi-locus and single marker gene (hetR) phylogenies of Trichodesmium. We used the following genomes: the four Trichodesmium MAGs from this study, five Trichodesmium MAGs from the TARA Oceans dataset $^{6}$ and that of Trichodesmium erythraeum IMS101 PRJNA318 ${ }^{18}$. The phylogenomic tree was constructed from a concatenated gene-alignment of a 251 single-copy gene-set hidden Markov Models (HMMs) for Cyanobacteria using GToTree (v.1.16.12; default settings $)^{38}$. The aligned protein sequences were refined using Gblocks $(0.91 \mathrm{~b}$; 
131 default settings) to eliminate poorly aligned positions and divergent regions ${ }^{39}$. A tree was

132 subsequently constructed from the cleaned alignment using IQtree2 with ModelFinder to estimate

133 the best-fit model (Q.plant $+\mathrm{F}+\mathrm{I}+\mathrm{G} 4)^{40}$. Shimodaira-Hasegawa approximate likelihood-ratio test

134 (SH-aLRT) and ultrafast bootstrap approximation (UFBoot) branch support values were estimated

135 from 1000 bootstraps. The tree was visualized using FigTree (v1.4.4) and rooted with Okeania

136 hirsuta (GCA_003838225) $)^{41}$ as an outgroup (Supplementary Table 4).

138 The hetR marker gene was identified in Trichodesmium MAGs using the Rapid Annotation using 139 Subsystem Technology (RAST) ${ }^{42}$. The hetR sequence and the neighboring genes cluster were 140 identified by searching for the HetR amino acid sequence (Tery_1921; Q93CE9) using BLAST on 141 the SEED server ${ }^{43}$. The marker gene sequences from each Trichodesmium MAG were aligned with 142 previously published ones ${ }^{16,17,44}$ and those from our Red Sea hetR amplicon sequencing using the 143 Multiple Alignment Fast Fourier Transformation (MAFFT; L-INS-i) software ${ }^{45}$. All the hetR 144 sequences used in this study can be found in Supplementary Table 5. This alignment was further 145 cleaned using Gblocks (0.91b; default settings; Castresana, 2000). A phylogenetic tree rooted at 146 the sequence of Okeania hirsuta, was constructed using IQtree2 for hetR (best-fit model 147 TPM3+F+I) and visualized using FigTree (Figure 3).

\section{Results and Discussion}

150 Four non-redundant Trichodesmium MAGs were assembled from the Red Sea (Figure 1;

151 Supplementary Table 1). The MAGs were of high quality, being 90\% complete and with less than 152 5\% redundancy, apart from MAG R04 which was 86.29\% complete. To provide a wider 153 framework of Trichodesmium diversity, we assembled three non-redundant Trichodesmium MAGs 
154 from the Pacific and the Atlantic Ocean, based on previously published raw data ${ }^{8,20,46}$. These 155 included two high-quality MAGs T01-2 and T02, as well as the lower quality MAG T01-1

156 (Supplementary Table 2).

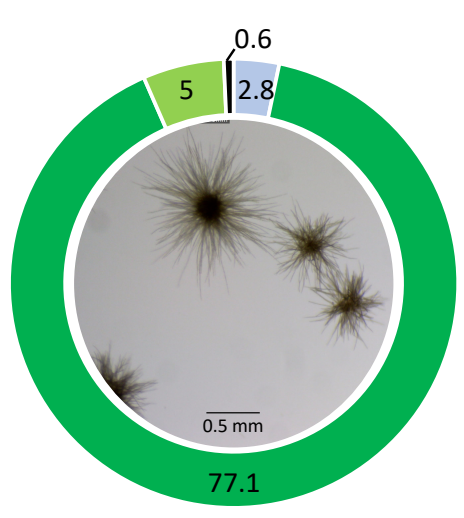

A. Dense puffs

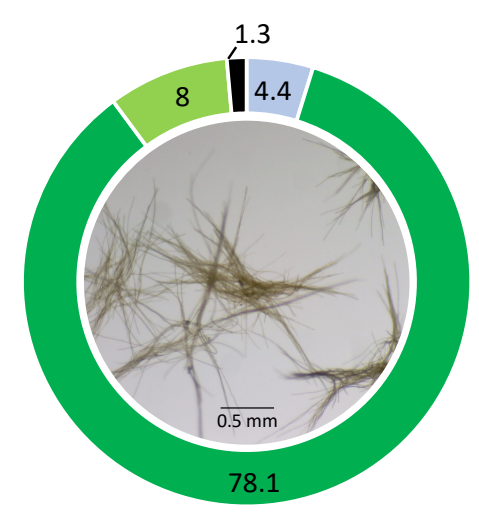

B. Thin puffs

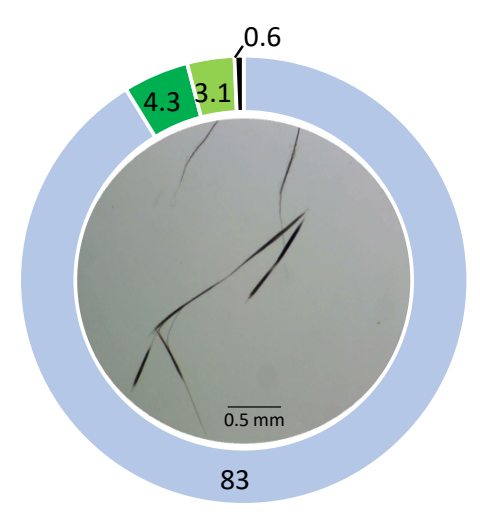

C. Tufts

\section{MAG ID - clade (hetR phylotype)}

Figure 1. Trichodesmium colony morphotypes and their relative read abundance (\%) in the Red Sea. Both (a) dense and (b) thin puff-shaped morphotypes, primarily consisted of T. thiebautii genotype (dark green; MAG R02), while tuft-shaped morphotypes (c) were dominated by T. erythraeum (light blue; MAG R01). All morphotypes contained low read abundance of non-diazotrophic MAG R03 (light green; MAG R03) and MAG R04 (black; MAG 
171 Both thin and dense puff-shaped colonies were dominated by $T$. thiebautii ( $78 \%$ read abundance)

172 (Table 1; Figure 1a, b). This finding is similar to another genomic study where radial (dense-like)

173 and non-radial puff morphologies were linked to the same genotype of T. thiebautii ${ }^{8}$. Puff-shaped

174 Trichodesmium colonies have the unique capability to interact, capture and concentrate dust at the

175 colony's core ${ }^{47}$ which can allow colonies to obtain limiting nutrients from the marine environment,

176 such as iron or phosphorous ${ }^{48,49}$. In the field, thin puffs isolated from the Red Sea were shown to

177 be more interactive in comparison to dense puffs in the capturing and concentrating of dust at the

178 colony's core, whilst exhibiting pronounced gliding motility ${ }^{7}$. Our findings highlight that these

179 morphological variations do not appear to be explained genomically and that other yet

180 uncharacterized factors are at play. We hypothesize that the observed variations in puff

181 morphologies concerning the centering of dust ${ }^{7,50}$ are due to differences in gene regulation in

182 response to yet unknown environmental conditions which may include nutrient limitation or

183 grazing. In a laboratory setting, single filaments of T. erythraeum IMS101 clustered into the puff

184 and tuft-shaped colonies when subjected to iron or phosphorous limitation ${ }^{51}$. Nonetheless,

185 Trichodesmium displays functional variability and heterogeneity at a single-colony level that remains largely unexplained ${ }^{9,10}$ and subsequently the ability to predict whether a colony will center

187 dust still requires ongoing exploration.

189 Our data revealed subspecies diversity for both $T$. thiebautii and T. erythraeum (Figure 2). T.

190 thiebautii MAGs appeared to cluster according to broad geographical patterns as MAG R02 was

191 more similar to that of T. thiebautii populations isolated from the Indian Ocean (98.3\% ANI), than

192 to the Atlantic T. thiebautii MAG (97.7\% ANI). This concurs with the previous findings ${ }^{6}$. We also

193 found genomic diversity within T. erythraeum as revealed from two distinct T. erythraeum MAGs 
194 from the Pacific Ocean (96.6\% ANI). MAG T01-1 was highly similar to that of T. erythraeum

195 isolated from the Red Sea (97.6\% ANI; MAG R01), whereas MAG T01-2 was closely related to

196 T. erythraeum IMS101 (99.4\% ANI), which was isolated from the Atlantic Ocean ${ }^{52}$. The hetR gene

197 phylotyping of Trichodesmium populations in the Red Sea indicates the occurrence of an additional

198 T. erythraeum subspecies similar to that of MAG T01-2 (Figure 3). We were, however, unable to

199 assemble its genome or link it to a specific morphotype. It is still unclear if these two T. erythraeum

200 subspecies within the Red Sea and the Pacific Ocean occupy distinct ecological niches.

\section{A. Phylogenetic tree}

B. ANI heatmap

$$
<95 \%
$$

$100 \%$ ANI

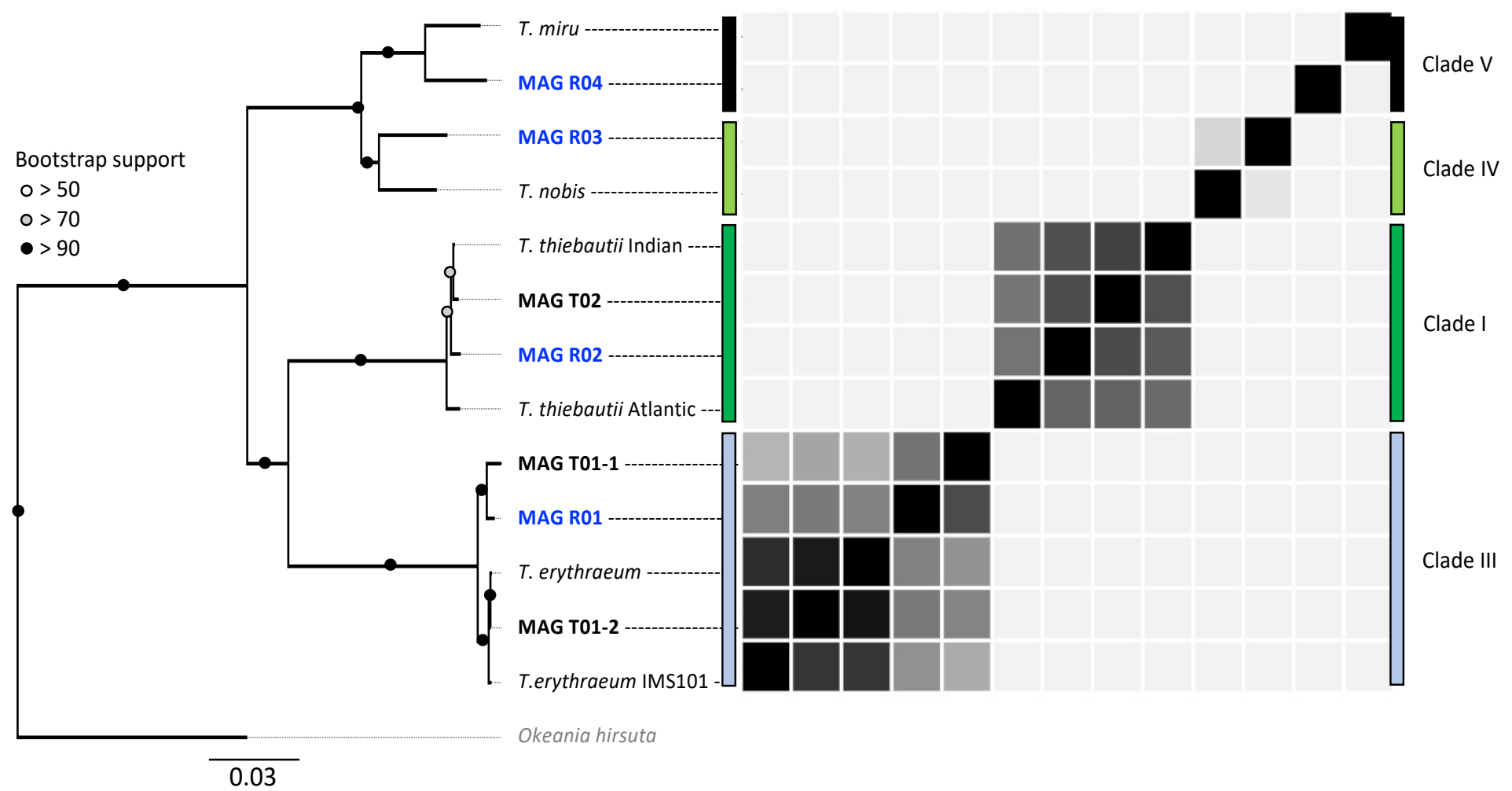

Figure 2. Multi-locus (251 HMMs) phylogenomic tree (a) and average nucleotide identity (ANI) heatmap (b) of Trichodesmium MAGs. All MAGs assembled in this study are marked in bold. Red Sea MAGs (our samples) are marked in blue. The phylogeny includes five MAGs from the TARA Oceans dataset and the laboratory culture $T$. erythraeum IMS101. The tree was rooted at Okeania hirsuta (grey). ANI values can be found in Supplementary Table 2 .

208 We detected two potentially non-diazotrophic species within the Red Sea with minor read 209 abundance in all three samples (Supplementary Table 1). Both genotypes could not be linked to a 
210 specific morphotype, and it is plausible that they occurred as single filaments within our samples.

211 The low coverage reflected a previous estimate of the non-diazotrophic Trichodesmium of the Red

212 Sea $^{6}$. Whereas MAG R03 clustered within clade IV, which includes the non-diazotrophic T. nobis

213 (95.6\% ANI), and MAG R04 clustered within clade V containing the non-diazotrophic T. miru

214 (94.6\% ANI), both MAGs are on the threshold of being a distinct species within clade IV and V,

215 respectively. We, therefore, turned to the single-marker gene het $R$ to further differentiate these

216 MAGs, as hetR phylogeny captures a larger diversity of Trichodesmium than is possible through

217 the limited number of Trichodesmium genomes currently available. The hetR sequences, but not

218 the genomes, are available for T. pelagicum (AF490696.1), T. hildebrandtii (AF490679.1) in clade

219 I, T. aureum (AF490680.1) in clade II, and T. contortum (AF013031.1), T. tenue (AF013033.1) in

220 clade IV and T. miru in clade V (Supplementary Table 4). 
Trichodesmium clades

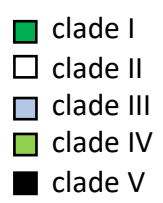

Bootstrap support

$0>50$

$0>70$

$\bullet>90$

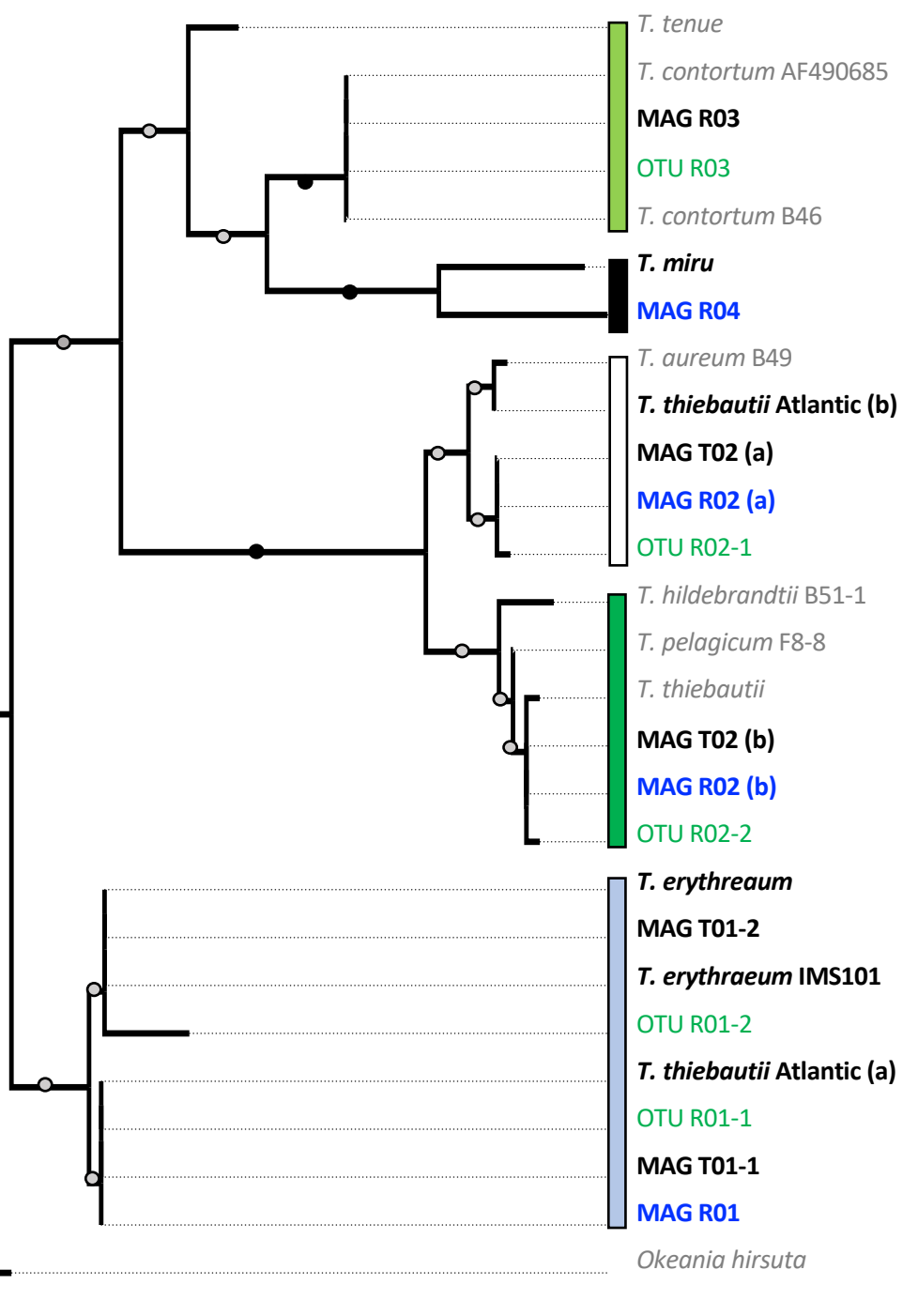

Figure 3. Trichodesmium hetR phylogeny. The tree is based on the alignment of 312 nucleotide sequences. Bold text indicates the hetR sequences from MAGs (Red Sea sequences are marked in blue). Amplicon het $R$ sequences are shown in green (Supplementary Table 4). The five proposed Trichodemisum clades are shown with colored boxes. Note that clades IV and V are polyphyletic and are therefore not completely resolved. MAGs R02 and T03 contain two het $R$ sequences, clustering within clades I and II, respectively. The hetR sequences from $T$. erythraeum genomes clustered into two groups within clade III. The percentage identity matrix between the het $R$ gene sequences can be found in Supplementary Table 3.

We show that $T$. nobis and $T$. contortum are closely related, potentially representing the same nondiazotrophic Trichodemsium species. Given that T. nobis has not been described morphologically, and the genome of $T$. contortum has not been sequenced, both can be linked through the phylogeny of marker genes. Whereas the previously published genome of T. nobis did not contain the hetR 
234 gene $^{6}$, the clade IV MAG R03 did, and its hetR gene sequence was $99.78 \%$ similar to that of $T$.

235 contortum (Figure 3; Supplementary Table 4). Complementing our findings, morphological

236 descriptions of $T$. contortum depict the species as single spiral-shaped trichomes, rather than

237 colonies, that are sporadically present in low abundance $(<1 \%$ of total Trichodesmium biomass)

238 within samples ${ }^{13,16,53}$. MAG R03 lacked the nitrogenase gene cluster, confirming that this clade is

239 non-diazotrophic.

240

241 Our results indicate that hetR phylotyping can overestimate the diversity within Trichodesmium

242 populations, in particular regarding clade II. Intriguingly, both T. thiebautii MAGs contained two

243 distinct hetR copies (906 bp), which had 28 (MAG R02) and 29 bp (MAG T02) single nucleotide

244 polymorphisms within a sequence. Further inspection of the two copies showed that they were

245 encoded in close vicinity of each other and that the synteny is highly conserved across all the

246 Trichodesmium genomes (Figure 4). These genes are likely paralogs of each other ${ }^{54}$ although it is

247 still unclear how $T$. thiebautii benefits from retaining both hetR sequences. Each hetR sequence

248 was placed into a distinct phylogenetic clade, where one copy grouped with the hetR sequence of

249 T. aureum (clade II), and the other one was placed within clade I, together with T. thiebautii, T.

250 pelagicum and T. hildebrandtii (Figure 3). Amplicon sequencing of the hetR gene showed highly

251 similar abundances of $T$. thiebautii and T. aureum in all the samples. Therefore, this is a likely

252 result of hetR paralog sequencing within a population of single $T$. thiebautii species

253 (Supplementary Figure 1). Alternatively, T. thiebautii and T. aureum may represent the

254 morphological diversity within the same genotype or species. We note that the Atlantic $T$.

255 thiebautii contained one hetR paralog that clustered with the hetR sequence of clade IV (T. 
257 to a single scaffold.

MAG R02 (T. thiebautii)

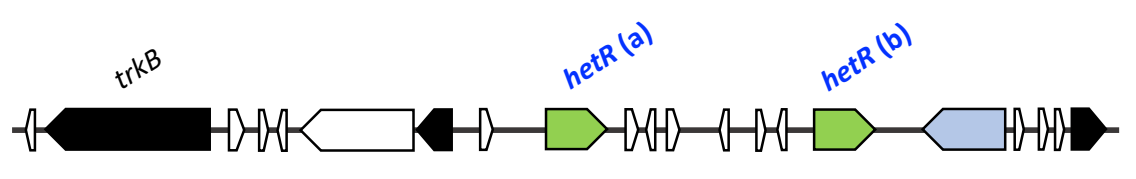

MAG R01 (T. erythraeum)

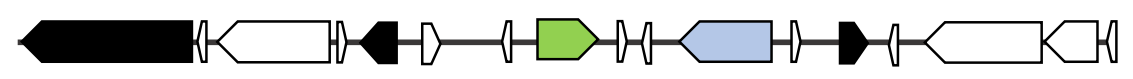

MAG R03 (T. contortum)

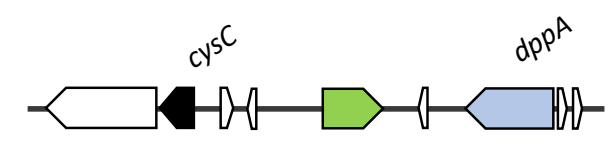

Biosynthesis gene

Trichodesmium erythraeum IMS101

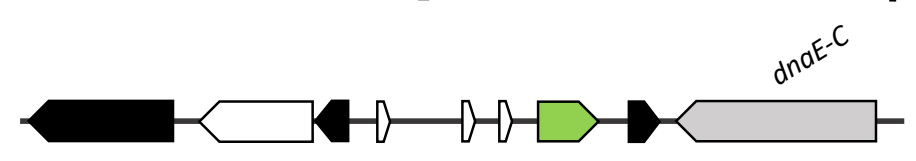

MAG R04 (T. miru)

Figure 4. The hetR gene clusters in Trichodesmium MAGs and T. erythraeum IMS101. The synteny of the hetR genes (green) and the neighboring genes is conserved. MAG R02 (T. thiebautii) contains two hetR genes (and are likely paralogs of each other).

Conclusions and Future Perspectives

264 Morphotype metagenomics enabled us to better capture the diversity that is present within

265 Trichodesmium populations while revisiting past attempts to differentiate between colonies ${ }^{55,56}$.

266 We were able to reconstruct the genomes of four different Trichodesmium species isolated from

267 the Red Sea, that were subsequently assigned to four different Trichodesmium clades including the

268 more elusive non-diazotrophic clades IV and V. We show that hetR gene-based phylotyping could

269 overestimate Trichodesmium diversity, highlighting the importance of re-assessing past attempts

270 at phylogeny. Overall, the phylogenetic and functional diversity of Trichodesmium in the world's 
271 oceans is still poorly understood to date. Thus, genomics in combination with gene expression

272 and physiology will remain an important avenue for future exploration of these key species.

273

274 Data Availability

275 The raw sequencing reads from the metagenomic study were deposited at NCBI under the

276 BioProject PRJNA804487. The bioinformatic analysis and scripts were compiled in an R

277 markdown file, together with the genomic FASTA files of the Trichodesmium MAGs constructed

278 for this paper, and are publicly available using the following GitHub repository link:

279 https://github.com/cocokoedooder/Trichodesmium_HetR/blob/main/README.md

280

281 Acknowledgments

282 This work was funded by the Israel Science Foundation $(260 / 21)$ and the Israel-USA Bi-National

283 Science Foundation (2020041) to YS. This work was also financially supported in part by the

284 Schulich Marine Studies Initiative to IBF. MRB acknowledges the support of the Israel Science

285 Foundation (913/19), the U.S.-Israel Binational Science Foundation (2019055) and the Israel

286 Ministry of Science and Technology (1126). FZ thanks the PBC Fellowship Program for

287 Outstanding Chinese and Indian Post-Doctoral Fellows. We would like to thank Murielle Dray for

288 technical support.

289

290 Competing Interests

291 The authors of this paper declare no conflict of interest. 


\section{Author Contributions}

CK, YS, IB-F and MR-B conceived the study. YS and IB-F acquired financial support. CK, FZ,

SB collected Trichodesmium colonies and extracted DNA for metagenomics. CK analyzed the metagenomic data under the guidance of MRB. EL performed the seasonal hetR amplicon

sequencing and data analysis under the guidance of IB-F. CK, EL and MR-B wrote the manuscript

with contributions from other co-authors. All authors discussed the results and revised the final

version of the manuscript.

301

\section{Bibliography}

3031 Transcription of National Library of Australia. Cook's Journal: Daily Entries, 22 August 1770. South Seas. 2004; 287.

2 Dugdale RC, Menzel DW, Ryther JH. Nitrogen fixation in the Sargasso Sea. DSR. 1961;

306 7: 297-300.

3 McKinna LIW. Three decades of ocean-color remote-sensing Trichodesmium spp. in the World's oceans: A review. Progress in Oceanography. 2015; 131: 177-199. significant marine cyanobacterium. Science 1997; 276: 1221-1229.

5 Capone DG. Coming full circle on diazotrophy in the marine cyanobacterium Trichodesmium. Proceedings of the National Academy of Sciences. 2021; 118: e2117967118.

6 Delmont TO. Discovery of nondiazotrophic Trichodesmium species abundant and widespread in the open ocean. Proceedings of the National Academy of Sciences. 2021; 118: e2112355118.

7 Wang S, Koedooder C, Zhang F, Kessler N, Eichner M, Shi D et al. Colonies of the marine cyanobacterium Trichodesmium optimize dust utilization by selective collection and retention of nutrient-rich particles. iScience. 2021; 25.

8 Gradoville MR, Crump BC, Letelier RM, Church MJ, White AE. Microbiome of Trichodesmium colonies from the North Pacific Subtropical Gyre. Frontiers in Microbiology. 2017; 8 .

9 Held NA, Sutherland KM, Webb EA, McIlvin MR, Cohen NR, Devaux AJ et al. Mechanisms and heterogeneity of in situ mineral processing by the marine nitrogen fixer Trichodesmium revealed by single-colony metaproteomics. ISME Communications. 2021; 1:35.

10 Eichner M, Basu S, Gledhill M, de Beer D, Shaked Y. Hydrogen dynamics in Trichodesmium colonies and their potential role in mineral iron acquisition. Frontiers in Microbiology. 2019; 10: 1565. 
11 Hansel CM, Buchwald C, Diaz JM, Ossolinski JE, Dyhrman ST, van Mooy BAS et al. Dynamics of extracellular superoxide production by Trichodesmium colonies from the Sargasso Sea. Limnology and Oceanography. 2016; 61: 1188-1200.

12 Rouco M, Haley ST, Dyhrman ST. Microbial diversity within the Trichodesmium holobiont. Environmental Microbiology. 2016; 18: 5151-5160.

13 Janson S, Siddiqui PJA, Walsby AE, Romans KM, Carpenter EJ, Bergman B. Cytomorphological of the planktonic diazotrophic Cyanobacteria Trichodesmium spp. from the Indian Ocaen and Caribbean and Sargasso Seas. Journal of Phycology. 1995; 31: 463-477.

14 McManus GB, Katz LA. Molecular and morphological methods for identifying plankton: what makes a successful marriage? Journal of Plankton Research. 2009; 31: 1119-1129.

15 Lundgren P, Janson S, Jonasson S, Singer A, Bergman B. Unveiling of novel radiations within Trichodesmium cluster by hetR gene sequence analysis. Applied and Environmental Microbiology. 2005; 71: 190-196.

16 Orcutt KM, Rasmussen U, Webb EA, Waterbury JB, Gundersen K, Bergman B. Characterization of Trichodesmium spp. by Genetic Techniques. Applied and Environmental Microbiology .2002; 68: 2236.

17 Hynes AM, Webb EA, Doney SC, Waterbury JB. Comparison of cultured Trichodesmium (Cyanophyceae) with species characterized from the field. Journal of Phycology. 2012; 48: 196-210.

18 Walworth N, Pfreundt U, Nelson WC, Mincer T, Heidelberg JF, Fu F et al. Trichodesmium genome maintains abundant, widespread noncoding DNA in situ, despite oligotrophic lifestyle. Proceedings of the National Academy of Sciences. 2015; 112: 42514256.

19 Frischkorn KR, Haley ST, Dyhrman ST. Coordinated gene expression between Trichodesmium and its microbiome over day-night cycles in the North Pacific Subtropical Gyre. ISME Journal. 2018; 12: 997-1007.

20 Frischkorn KR, Rouco M, van Mooy BAS, Dyhrman ST. Epibionts dominate metabolic functional potential of Trichodesmium colonies from the oligotrophic ocean. The ISME Journal. 2017 11:9 2017; 11: 2090-2101.

21 Kieser S, Brown J, Zdobnov EM, Trajkovski M, McCue LA. ATLAS: A Snakemake workflow for assembly, annotation, and genomic binning of metagenome sequence data. BMC Bioinformatics. 2020; 21: 1-8.

22 B. Bushnell. BBTools software package. sourceforge.net/projects/bbmap/ (accessed 16 Dec 2021).

23 Bushnell B, Rood J, Singer E. BBMerge - Accurate paired shotgun read merging via overlap. PLOS ONE.. 2017; 12: e0185056.

24 Nurk S, Meleshko D, Korobeynikov A, Pevzner PA. metaSPAdes: a new versatile metagenomic assembler. Genome Research. 2017; 27: 824-834.

25 Kang DD, Li F, Kirton E, Thomas A, Egan R, An H et al. MetaBAT 2: An adaptive binning algorithm for robust and efficient genome reconstruction from metagenome assemblies. PeerJ. 2019; 2019: e7359.

26 Wu YW, Simmons BA, Singer SW. MaxBin 2.0: an automated binning algorithm to recover genomes from multiple metagenomic datasets. Bioinformatics. 2016; 32. 
27 Nissen JN, Johansen J, Allesøe RL, Sønderby CK, Armenteros JJA, Grønbech CH et al. Improved metagenome binning and assembly using deep variational autoencoders. Nature biotechnology. 2021; 39: 555-560. quality of microbial genomes recovered from isolates, single cells, and metagenomes. Genome Research. 2015; 25: 1043-1055.

29 Sieber CMK, Probst AJ, Sharrar A, Thomas BC, Hess M, Tringe SG et al. Recovery of genomes from metagenomes via a dereplication, aggregation and scoring strategy. Nature Microbiology. 2018 3:7 2018; 3: 836-843.

30 Olm MR, Brown CT, Brooks B, Banfield JF. dRep: a tool for fast and accurate genomic comparisons that enables improved genome recovery from metagenomes through dereplication. The ISME Journal. 2017 11:12 2017; 11: 2864-2868.

31 Parks DH, Chuvochina M, Waite DW, Rinke C, Skarshewski A, Chaumeil PA et al. A standardized bacterial taxonomy based on genome phylogeny substantially revises the tree of life. Nature Biotechnology. 2018 36:10 2018; 36: 996-1004.

32 Hyatt D, Chen GL, LoCascio PF, Land ML, Larimer FW, Hauser LJ. Prodigal: Prokaryotic gene recognition and translation initiation site identification. $B M C$ Bioinformatics. 2010; 11: 1-11.

33 Pritchard L, Glover RH, Humphris S, Elphinstone JG, Toth IK. Genomics and taxonomy in diagnostics for food security: soft-rotting enterobacterial plant pathogens. Analytical Methods. 2015; 8: 12-24.

34 Massana R, Murray AE, Preston CM, DeLong EF. Vertical distribution and phylogenetic characterization of marine planktonic Archaea in the Santa Barbara Channel. Applied and Environmental Microbiology. 1997; 63: 50. High-resolution sample inference from Illumina amplicon data. Nature Method. 2016 13:7 2016; 13: 581-583.

40136 Bolyen E, Rideout JR, Dillon MR, Bokulich NA, Abnet CC, Al-Ghalith GA et al. Reproducible, interactive, scalable and extensible microbiome data science using QIIME 2. Nature Biotechnology 2019. 37:8 2019; 37: 852-857.

37 Rognes T, Flouri T, Nichols B, Quince C, Mahé F. VSEARCH: a versatile open source tool for metagenomics. PeerJ. 2016; 4.

38 Lee MD. GToTree: a user-friendly workflow for phylogenomics. Bioinformatics. 2019; 35: 4162-4164.

39 Castresana J. Selection of conserved blocks from multiple alignments for their use in phylogenetic analysis. Molecular Biology and Evolution. 2000; 17: 540-552.

40 Minh BQ, Schmidt HA, Chernomor O, Schrempf D, Woodhams MD, von Haeseler A et al. IQ-TREE 2: New models and efficient methods for phylogenetic inference in the genomic era. Molecular Biology and Evolution. 2020; 37: 1530-1534. Ketoreductase Domain Dysfunction Expands Chemodiversity: Malyngamide Biosynthesis in the cyanobacterium Okeania hirsuta. ACS chemical biology. 2018; 13: 3385-3395. 
43 Aziz RK, Devoid S, Disz T, Edwards RA, Henry CS, Olsen GJ et al. SEED servers: highperformance access to the SEED genomes, annotations, and metabolic models. PloS one. 2012; 7: e48053.

44 Janson S, Bergman B, Carpenter EJ, Giovannoni SJ, Vergin K. Genetic analysis of natural populations of the marine diazotrophic cyanobacterium Trichodesmium. FEMS Microbiology Ecology. 1999; 30: 57-65.

45 Katoh K, Kuma KI, Toh H, Miyata T. MAFFT version 5: improvement in accuracy of multiple sequence alignment. Nucleic Acids Research. 2005; 33: 511-518. Trichodesmium physiological ecology and phosphate reduction in the western tropical South Pacific. Biogeoscience.s 2018; 15: 5761-5778.

47 Rubin M, Berman-Frank I, Shaked Y. Dust-and mineral-iron utilization by the marine dinitrogen-fixer Trichodesmium. Nature Geoscience 2011; 4: 529-534.

48 Basu S, Shaked Y. Mineral iron utilization by natural and cultured Trichodesmium and associated bacteria. Limnology and Oceanography. 2018; 63: 2307-2320.

49 Basu S, Gledhill M, de Beer D, Prabhu Matondkar SG, Shaked Y. Colonies of marine cyanobacteria Trichodesmium interact with associated bacteria to acquire iron from dust. Communications Biology. 2019; 2: 1-8.

50 Kessler N, Armoza-Zvuloni R, Wang S, Basu S, Weber PK, Stuart RK et al. Selective collection of iron-rich dust particles by natural Trichodesmium colonies. The ISME Journal. 2019; 14: 91-103.

51 Tzubari Y, Magnezi L, Be'Er A, Berman-Frank I. Iron and phosphorus deprivation induce sociality in the marine bloom-forming cyanobacterium Trichodesmium. ISME Journal.

52 Prufert-Bebout L, Paerl HW, Lassen C. Growth, nitrogen Fixation, and spectral

444

445

446 attenuation in cultivated Trichodesmium species. Applied and Environmental Microbiology 1993; 59: 1367-1375.

53 Letelier RM, Karl DM. Role of Trichodesmium spp. in the productivity of the subtropical North Pacific Ocean. Marine Ecology Progress Series 1996; 133: 263-273.

54 Koonin E v. Orthologs, paralogs, and evolutionary genomics. Annual Review of Genetics. 2005; 39: 309-338.

450

451

55 Scornavacca C, Delsuc F, Galtier N (eds.). Phylogenetics in the Genomic Era. 2020

56 Smith ML, Hahn MW. New Approaches for inferring phylogenies in the presence of

453 paralogs. Trends in Genetics. 2021; 37: 174-187. 


\section{Supplementary Information}

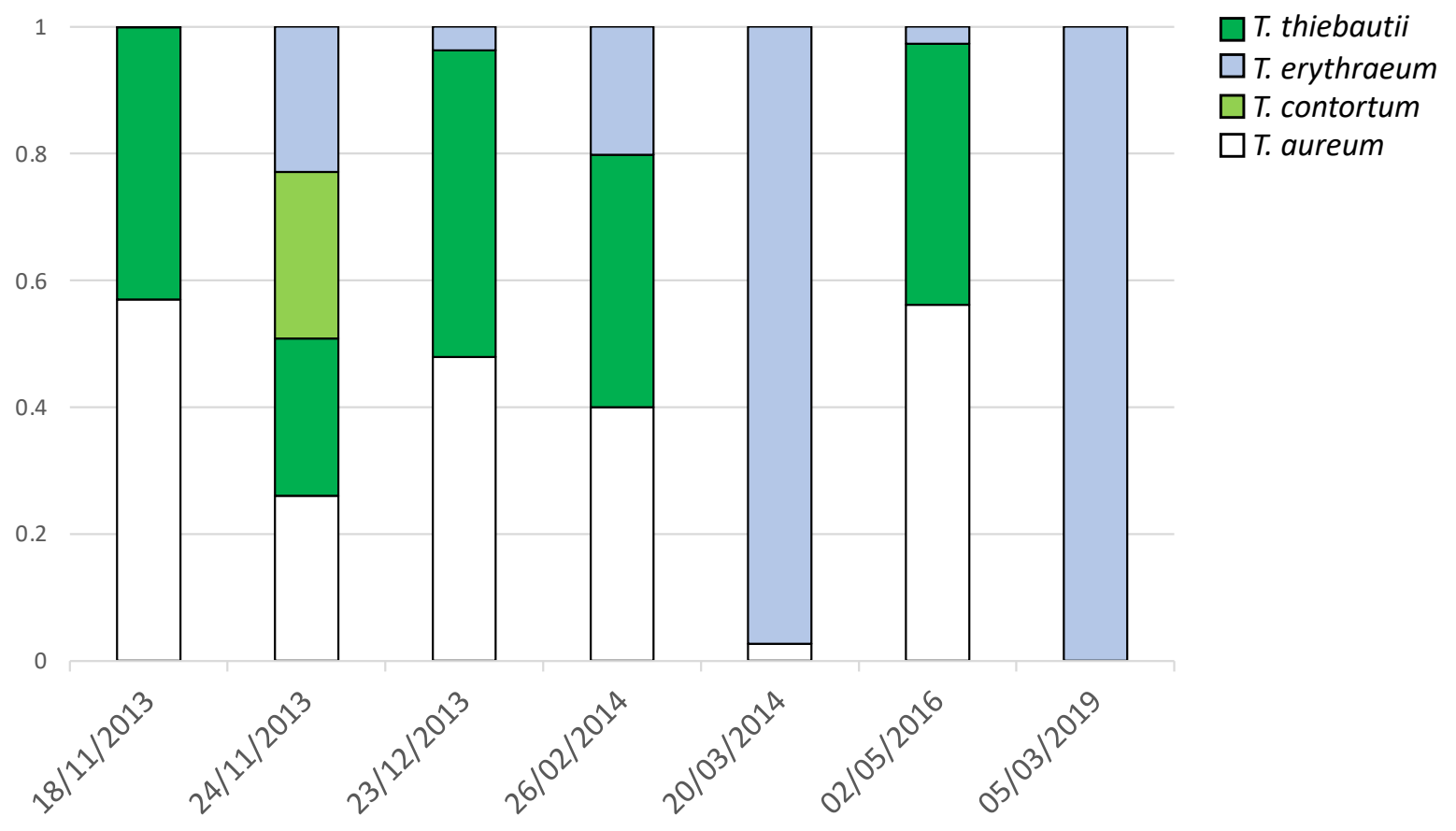

461

462 Supplementary Figure 1. Displaying the relative abundance of hetR sequences from (puff and tuft)

463 Trichodesmium colonies isolated from the Red Sea. Note that T. thiebautii and T. aureum are in similar proportions 464 to each other. The presence of $T$. aureum is likely an artifact and instead represents a paralog het $R$ sequence present 465 in T. thiebautii, thereby effectively over-estimating Trichodesmium diversity within the sample.

468 Supplementary Table 1. General information about the Trichodesmium MAGs derived from this study.

\begin{tabular}{|l|l|l|c|c|c|c|c|c|c|}
\hline MAG ID & Accession Number & HetR phylotype & Size (Mbp) & Completeness & Redundancy & A. Dense-puff (\%) & B. Long-puff (\%) & C. Tuft (\%) \\
\hline MAG R01 & SAMN25809967 & T. erythreaum & 6.641 & 95.8 & 0.3 & 2.8 \\
\hline MAG R02 & SAMN25809968 & T. thiebautii/ T. aureum & 6.769 & 94.8 & 0.4 & 7.4 \\
\hline MAG R03 & SAMN25809969 & T. nobis & 5.841 & 94.0 & 2.5 & 7.1 \\
\hline MAG R04 & SAMN25809970 & T. contortum & 4.176 & 83.0 & 3.1 & 5 \\
\hline
\end{tabular}

